\title{
Signos vasculares retinianos en recién nacidos con cardiopatía congénita crítica: serie de casos
} Retinovascular findings in newborns with critical congenital heart disease: A case series

\author{
Dra. Nurdan Fettah, ${ }^{a}$ Dr. Emrah U. Kabatas, ${ }^{b}$ Dr. Vehbi Doğan, ${ }^{c}$ Prof. Asoc. Dra. Aysegül Zenciroğlu, ${ }^{a}$ \\ Prof. Asoc. Dra. Dilek DilliI, ${ }^{a}$ Dra. Elif Özyazıcı, ${ }^{a}$ Dr. Murat Koç, ${ }^{d}$ Prof. Asoc. Dr. Serdar Beken, ${ }^{a}$ \\ Prof. Asoc. Dra. Arzu Dursun y Dra. Selmin Karademir
}

\begin{abstract}
RESUMEN
La cardiopatía congénita crítica (CCC) podría provocar cambios en la vasculatura de la retina. Sin embargo, no se dispone de suficientes datos sobre este problema en los recién nacidos. Se evaluaron los cambios en la vasculatura retiniana en una serie de 43 recién nacidos con CCC. Se los dividió en dos grupos según el tipo de CCC; grupo $1(\mathrm{n}=18)$ : CCC obstructiva izquierda y grupo $2(n=25)$ : CCC obstructiva derecha. Se detectaron enfermedades vasculares retinianas en 21 pacientes $(48,8 \%)$; estas fueron más frecuentes en el grupo $1(\mathrm{p}=0,04)$. La patología más común fue la tortuosidad vascular retiniana, observada en seis pacientes $(33,3 \%)$ del grupo 1 y en $4(16,0 \%)$ del grupo 2 . Ninguno de los 21 pacientes con cambios en la vasculatura de la retina requirió tratamiento durante el seguimiento. En un análisis multivariado, solo la CCC obstructiva izquierda estuvo asociada con el desarrollo de enfermedad vascular retiniana $(\mathrm{P}=0,03$, razón de probabilidades [OR]:2,8, intervalo de confianza [IC] del 95\%: 1,1-7,4). Los cambios vasculares retinianos son frecuentes en los recién nacidos con CCC.

Palabras clave: defectos cardíacos congénitos, anomalías en los vasos retinianos, recién nacidos.
\end{abstract}

http:/ / dx.doi.org/10.5546/ aap.2017.e175

Texto completo en inglés:

http: / / dx.doi.org/10.5546/ aap.2017.eng.e175

Cómo citar: Fettah N, Kabatas EU, Doğan V, et al. Signos vasculare retinianos en recién nacidos con cardiopatía congénita crítica: serie de casos. Arch Argent Pediatr 2017;115(3):e175-e178.

a. Departamento de Neonatología.

b. Departamento de Oftalmología.

c. Departamento de Cardiología Pediátrica.

d. Departamento de Cirugía Cardiovascular Pediátrica.

Hospital de Formación e Investigación Materno Infantil

Dr. Sami Ulus, Ankara, Turquía.

Correspondencia:

Dra. Dilek Dilli: dilekdilli2@yahoo.com

Financiamiento: Ninguno.

Conflicto de intereses: Ninguno que declarar.

Recibido: 2-10-2016

Aceptado: 27-12-2016

\section{INTRODUCCIÓN}

La cardiopatía congénita (CC) es una anomalía en la estructura o función del sistema cardiovascular, presente al momento del nacimiento, aunque la enfermedad suele diagnosticarse en el período posnatal. Con la etiopatogenia de la $\mathrm{CC}$, se han relacionado factores tanto genéticos como ambientales. ${ }^{1} \mathrm{Se}$ ha informado que la CC afecta a 8/1000 recién nacidos. ${ }^{2}$ Alrededor del $25 \%$ de los bebés con CC tienen CC crítica (CCC) que suele requerir cirugía $\mathrm{u}$ otros procedimientos durante el primer año de vida. ${ }^{2,3}$

Según diversos informes, la CC podría tener efectos sobre la vasculatura de la retina. ${ }^{4-6}$ Hasta donde sabemos, no se han realizado investigaciones sobre los cambios vasculares retinianos en los recién nacidos con CCC. Nuestro objetivo fue determinar los signos vasculares retinianos en los recién nacidos con CCC.

\section{MATERIALES Y MÉTODOS}

En esta serie de casos, se incluyó a 43 pacientes recién nacidos a término o cerca de término con CCC internados en la unidad de cuidados intensivos neonatales (UCIN) de un hospital de alta complejidad entre mayo de 2013 y diciembre de 2014. Se excluyó a los recién nacidos con $<35$ semanas de gestación o con una anomalía congénita importante. Tampoco se incluyó a los recién nacidos cuyos padres no otorgaron el consentimiento. El diagnóstico de CCC se estableció por ecocardiograma o cateterismo cardíaco a cargo de un cardiólogo pediátrico. Se definió CCC como la CC que requería intervención quirúrgica o cardíaca por catéter. Se evaluó el puntaje de riesgo de la intervención según el ajuste del riesgo en cirugía de cardiopatía congénita (Risk Adjustment in Congenital Heart Surgery, RACHS-1). ${ }^{7}$

El mismo oftalmólogo realizó las evaluaciones oculares en la primera semana de hospitalización en la UCIN. Las evaluaciones oculares se repitieron semanalmente en caso de que se 
observaran cambios vasculares retinianos. Se definió como retraso de la vascularización retiniana la ausencia de vasos retinianos en la ora serrata temporal después de las 40 semanas de edad gestacional.

Se registraron las concentraciones máximas y mínimas del hematocrito (Hto.; \%), la presión parcial de oxígeno máxima y mínima $\left(\mathrm{PaO}_{2} ; \mathrm{mmHg}\right)$ obtenida a partir de gasometrías y la cantidad de transfusiones de glóbulos rojos. Se registraron también la duración del uso de respirador, de presión positiva continua (PPC) nasal y la duración de exposición al oxígeno libre y total.

Se dividió a los pacientes en dos grupos; grupo 1: CCC obstructiva izquierda y grupo 2: CCC obstructiva derecha. Se comparó a los grupos en términos de características clínicas y signos vasculares retinianos.

El comité de ética local aprobó el estudio. Se proporcionó información completa sobre la naturaleza y el objetivo del estudio a los padres, quienes brindaron su consentimiento por escrito.

\section{Análisis estadísticos}

Los datos se analizaron en una computadora con el paquete estadístico SPSS 18.0 (SPSS Inc. Chicago, Illinois, EE. UU.). Los datos paramétricos se expresaron como media \pm desviación estándar (DE) y los datos no paramétricos, como mediana e intervalo intercuartílico (IIC). Las diferencias entre los grupos se evaluaron con la prueba $t$ de Student o la prueba U de Mann-Whitney, según correspondiera. Se utilizó la prueba $\chi^{2}$ para los datos categóricos. Se realizó un análisis multivariado para definir los factores asociados con la presencia de enfermedad vascular retiniana en los pacientes con CCC. La edad gestacional, el tipo de CCC, la duración de la exposición al oxígeno total, la cantidad de transfusiones de glóbulos rojos, las concentraciones máxima y mínima de Hto. y $\mathrm{PaO}_{2}$ se consideraron variables independientes. El nivel de significancia se estableció en 0,05 para todas las comparaciones.

TABla 1. Características demográficas y clínicas de los pacientes del estudio, $N=43$

\begin{tabular}{|c|c|c|}
\hline & Grupo $1(n=18)$ & Grupo $2(n=25)$ \\
\hline Edad gestacional (semanas), media $\pm \mathrm{DE}$ & $38,4 \pm 1,4$ & $37,7 \pm 1,3$ \\
\hline Peso al nacer $(\mathrm{g})$, media $\pm \mathrm{DE}$ & $3332 \pm 366$ & $2992 \pm 650$ \\
\hline Índice de parto por cesárea, n (\%) & $8(53,3)$ & $7(28)$ \\
\hline Sexo masculino, $\mathrm{n}(\%)$ & $11(64,7)$ & $14(56)$ \\
\hline Diagnóstico prenatal, $\mathrm{n}(\%)$ & $1(5,6)$ & $3(12,0)$ \\
\hline Edad al momento de la hospitalización, mediana (IIC) & $6(3-9,5)$ & $6(1,5-10)$ \\
\hline Puntaje RACHS-1, mediana (IIC) & $2(2-6)$ & $3(2-3)$ \\
\hline Patología vascular retiniana, $\mathrm{n}(\%)$ & $12(66,7)$ & $9(36)$ \\
\hline Retraso de la vascularización & $2(11,1)$ & $5(20)$ \\
\hline Aumento de la tortuosidad vascular retiniana & $6(33,3)$ & $4(16)$ \\
\hline Hemorragia retiniana & $1(5,6)$ & 0 \\
\hline Retinopatía del prematuro & $3(16,7)$ & 0 \\
\hline Duración de la exposición al oxígeno total (días), mediana (IIC) & $23(18-33)$ & $25(12-49)$ \\
\hline Respirador & $7(4,5-21)$ & $15(5-23)$ \\
\hline PPC nasal & $4(2,5-5,5)$ & $5(4-14)$ \\
\hline Oxígeno libre & $10(7-15)$ & $10,5(4-24)$ \\
\hline Cantidad de transfusiones de glóbulos rojos, mediana (IIC) & $2(1-3)$ & $3(1-4)$ \\
\hline \multicolumn{3}{|l|}{$\mathrm{PaO}_{2}(\mathrm{mmHg})$, mediana (IIC) } \\
\hline $\mathrm{PaO}_{2}$ máxima & $85(72-91)$ & $85(76-91)$ \\
\hline $\mathrm{PaO}_{2}$ mínima & $43(32-58)$ & $40(35-55)$ \\
\hline \multicolumn{3}{|l|}{ Hto. (\%),mediana (IIC) } \\
\hline $\mathrm{PaO}_{2}$ máxima & $45(40-48)$ & $45(41-49)$ \\
\hline $\mathrm{PaO}_{2}$ mínima & $30(27-33)$ & $29(28-34)$ \\
\hline Hospitalización (días), mediana (IIC) & $28(20-40)$ & $33(26-90)$ \\
\hline Mortalidad, n $(\%)$ & $8(44,4)$ & $7(28,0)$ \\
\hline Inversión del cromosoma 9 & $1(5,6)$ & - \\
\hline Monosomía X & - & $1(0,4)$ \\
\hline
\end{tabular}

Grupo 1: cardiopatía congénita crítica obstructiva izquierda. Grupo 2: cardiopatía congénita crítica obstructiva derecha. Hto: hematocrito, $\mathrm{PaO}_{2}$ : presión parcial de oxígeno, RACHS-1: ajuste del riesgo en cirugía de cardiopatía congénita (Risk Adjustment in Congenital Heart Surgery). 


\section{RESULTADOS}

En relación con todos los pacientes, la media de la edad gestacional y del peso al nacer fueron $38,0 \pm 1,4$ semanas y $3134 \pm 570 \mathrm{~g}$, respectivamente. En el grupo 1 había 18 pacientes: estenosis/atresia de la válvula aórtica, $\mathrm{n}=3$; interrupción del cayado aórtico, $\mathrm{n}=2$; coartación aórtica, $\mathrm{n}=11$; síndrome del corazón izquierdo hipoplásico, $\mathrm{n}=2$. En el grupo 2, había 25 pacientes: estenosis / atresia pulmonar, $\mathrm{n}=23$; tetralogía de Fallot $(\mathrm{TF})$, $\mathrm{n}=2$. Las características demográficas y clínicas de los pacientes del estudio se describen en la Tabla 1.

Se detectaron enfermedades vasculares retinianas en 21 pacientes (48,8\%); estas fueron más frecuentes en el grupo $1(P=0,04)$. La tortuosidad vascular retiniana fue la patología más común, observada en seis pacientes $(33,3 \%)$ del grupo 1 y en 4 (16\%) del grupo 2 (Figura 1). Se observó retraso de la vascularización de la retina temporal en dos pacientes $(11,1 \%)$ del grupo 1 y en cinco $(20,0 \%)$ del grupo 2 . La media de la edad gestacional de estos recién nacidos fue $37,1 \pm 0,9$ semanas. Conforme a las evaluaciones oculares semanales, se determinó que la vascularización completa de la retina ocurría a una media de edad desde la fecundación de $46,3 \pm 1,6$ semanas.

En el grupo 1, se detectó retinopatía del prematuro en estadio 1 en tres pacientes nacidos a término o cerca de término con coartación aórtica; la media de su edad gestacional y del peso al nacer fueron $36,6 \pm 0,5$ semanas y $3463 \pm 686 \mathrm{~g}$,

FIgURA 1. Aumento de la tortuosidad vascular retiniana en un paciente con coartación aórtica

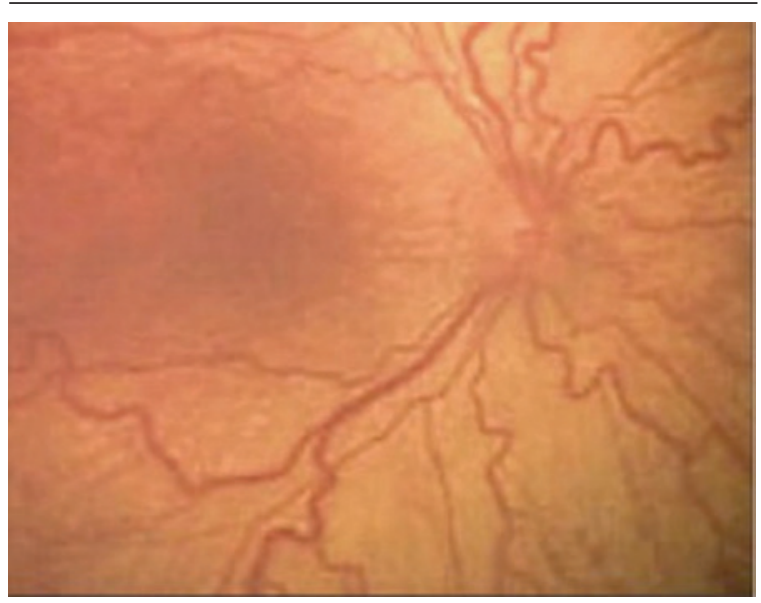

respectivamente. Los tres pacientes nacieron en un centro sanitario dotado de UCIN, a la cual fueron ingresados inmediatamente después del parto. Por lo tanto, las primeras evaluaciones oculares se realizaron en la primera semana de vida. Se determinó la presencia de retinopatía durante la segunda evaluación ocular una semana después de la primera. En el mismo grupo, se detectó hemorragia retiniana en un paciente $(5,6 \%)$, que también tenía hemorragia intracraneal de grado 2. En un análisis multivariado, solo la CCC obstructiva izquierda estuvo asociada con la presencia de una enfermedad vascular retiniana $(P=0,03$, razón de probabilidades [OR]: 2,8, intervalo de confianza [IC] del 95\%: 1,1-7,4).

La evaluación citogenética con bandeo cromosómico de Giemsa (GTG) reveló monosomía $\mathrm{X}$ en una paciente con coartación aórtica que tenía características fenotípicas del síndrome de Turner. La coartación se reparó con resección y anastomosis término-terminal. Se detectó inversión del cromosoma 9 en dos pacientes con atresia pulmonar. Estos pacientes nacieron de padres no consanguíneos y no tenían ninguna otra anomalía clínica. Se los sometió a una derivación de Blalock Taussig (BT).

Se realizó una derivación de BT en 23 pacientes $(53,5 \%)$, reparación de la coartación con resección y anastomosis término-terminal en $13(30,2 \%)$, valvuloplastía con balón en 3 $(7,0 \%)$, cirugía de Norwood en $2(4,7 \%)$ y ablación por radiofrecuencia en $2(4,7 \%)$. La tasa global de mortalidad fue del 34,9\% ( $n=15)$. De los 21 pacientes $(33,3 \%)$ con patología vascular retiniana, siete murieron a causa de la CCC preexistente o de las complicaciones asociadas. Entre los recién nacidos supervivientes, la retinopatía y la hemorragia retiniana se resolvieron espontáneamente dentro de las cinco semanas posteriores a la detección inicial. La tortuosidad vascular retiniana se resolvió aproximadamente tres meses después de la cirugía cardíaca.

\section{DISCUSIÓN}

En esta serie de casos, se observó que los recién nacidos con CCC presentan diversas enfermedades vasculares retinianas. Si bien se desconoce el mecanismo preciso de estos cambios, lo más probable es que la interacción de factores complejos, tales como el aumento de la viscosidad y la hipoxemia, participen en la patogenia. ${ }^{8-9}$

Los signos oculares más frecuentemente informados en los pacientes con CC cianótica 
son tortuosidad vascular retiniana, hemorragia retiniana, edema del disco óptico, papiledema, retinopatía isquémica, uveítis y obstrucción de la vena central de la retina. ${ }^{4-6}$ En otros informes de casos, se observaron diversos cambios vasculares y retinopatía con CC cianótica. ${ }^{10-12}$ Entre estas, la tortuosidad vascular retiniana parece ser el cambio oftalmológico más frecuente en los pacientes con $\mathrm{CC}$, al igual que en nuestra serie de casos.

Los factores de riesgo más importantes para el desarrollo de retinopatía del prematuro son una menor edad gestacional ( $<32$ semanas) y un bajo peso al nacer $(<1500 \mathrm{~g}) \cdot{ }^{13} \mathrm{Sin}$ embargo, se detectó retinopatía en estadio 1 en tres pacientes nacidos cerca de término con coartación aórtica. Esta última podría contribuir al desarrollo de retinopatía en los pacientes nacidos a término o cerca de término en las primeras semanas de vida.

La retina nasal suele estar totalmente vascularizada después de los ocho meses de gestación mientras que la retina periférica y temporal, hacia el mes después del parto de los recién nacidos a término y sanos. ${ }^{13}$ En nuestra serie, se observó retraso de la vascularización de la retina temporal en siete pacientes. Puede considerarse que la CC podría ser la causa del retraso en la maduración vascular de la retina.

Peterson y Rosenthal ${ }^{14}$ informaron que la gravedad de los cambios del fondo estuvo asociada con la saturación de oxígeno arterial y el hematocrito del paciente. De manera similar, Mansour y col. ${ }^{4}$ identificaron dilatación vascular retiniana y aumento de la tortuosidad vascular en los pacientes con CC. En nuestro estudio, el análisis multivariado mostró que solo la CCC obstructiva izquierda estuvo asociada con el desarrollo de una enfermedad vascular retiniana.

Tsui y col. ${ }^{10}$ describieron el aumento de la tortuosidad vascular retiniana en cuatro adultos asintomáticos con CC cianótica. Observaron que la elevación de la saturación de oxígeno después de la cirugía disminuyó la hipoxemia y la eritrocitosis, con normalización de los patrones de vascularidad retiniana. De manera similar, observamos que la tortuosidad vascular retiniana se resolvió aproximadamente tres meses después de la cirugía cardíaca.

El alto porcentaje de signos oculares en la CC podría estar relacionado con la incidencia elevada de síndromes asociados. ${ }^{4}$ En la población de nuestro estudio, se detectó inversión del cromosoma 9 en dos pacientes con atresia pulmonar y síndrome de Turner en un paciente con coartación aórtica.
Si bien, en esta serie de casos, se investigaron los cambios vasculares retinianos en el período neonatal de los pacientes con CCC, una de las limitaciones del estudio es la pequeña cantidad de pacientes con CCC; como resultado, es posible que el poder estadístico no haya sido suficiente para detectar otras enfermedades oculares. Por otro lado, no fue posible evaluar los cambios vasculares retinianos en los pacientes fallecidos. Aun así, hasta donde sabemos, este es el primer estudio sobre cambios vasculares retinianos en recién nacidos con CCC.

A modo de conclusión, la CCC podría causar cambios vasculares retinianos en los recién nacidos, aunque estos no siempre requirieron tratamiento. La función de los oftalmólogos parece ser más relevante en este campo.

\section{REFERENCIAS}

1. Huang JB, Liu YL, Lv XD. Pathogenic mechanisms of congenital heart disease. Fetal Pediatr Pathol 2010;29(5):359-72.

2. Strauss A, Toth B, Schwab B, Fuchshuber S, et al. Prenatal diagnosis of congenital heart disease and neonatal outcome--a six years experience. Eur J Med Res 2001;6(2):66-70.

3. Gilboa SM, Salemi JL, Nembhard WN, Fixler DE, et al. Mortality resulting from congenital heart disease among children and adults in the United States, 1999 to 2006. Circulation 2010;122(22):2254-63.

4. Mansour AM, Bitar FF, Traboulsi EI, Kassak KM, et al. Ocular pathology in congenital heart disease. Eye (Lond) 2005;19(1):29-34.

5. Crowe RJ, Kohner EM, Owen SJ, Robinson DM. The retinal vessels in congenital heart disease. Med Biol Illus1969;19(2):95-9.

6. Tan H, Ceviz N, Baykal O, Büyükavci M, et al. Pulmonary atresia/ventricular septal defect associated with facial portwine stain and retinal vascular abnormality: a new constellation? Am J Med Genet A 2003;122A(3):266-8.

7. Jenkins KJ, Gauvreau K, Newburger JW, Spray TL, et al. Consensus-based method for risk adjustment for surgery for congenital heart disease. J Thorac Cardiovasc Surg 2002;123(1):110-8.

8. Cordina RL, Celermajer DS. Chronic cyanosis and vascular function: implications for patients with cyanotic congenital heart disease. Cardiol Young 2010;20(3):242-53.

9. DeSchweinitzGE, Woods AC. Concerning the ocular symptoms of erythremia (chronic polycythemia vera), with special reference to the fundus picture. Trans Am Ophthalmol Soc 1925;23:90-105.

10. Tsui I, Shamsa K, Perloff JK, Lee E, et al. Retinal vascular patterns in adults with cyanotic congenital heart disease. Semin Ophthalmol 2009;24(6):262-5.

11. Ahmad OF, Hirose T. Severe retinopathy in a child with hypoplastic left heart syndrome. Am J Ophthalmol 2004;137(3):566-7.

12. El-Asrar AM, Awad A, Tabbara KF. Retinal arterial macroaneurysm in a patient with congenital heart disease. $\mathrm{Br}$ J Ophthalmol 1993;77(9):606-7.

13. Section on Ophthalmology American Academy of Pediatrics; American Academy of Ophthalmology; American Association for Pediatric Ophthalmology and Strabismus. Screening examination of premature infants for retinopathy of prematurity. Pediatrics 2006; 117(2):572-6.

14. Petersen RA, Rosenthal A. Retinopathy and papilledema in cyanotic heart disease. Pediatrics 1972;49(2):243-9. 